\title{
SENSITIVITY AND SPECIFICITY OF VISUAL INSPECTION WITH LUGOL'S IODINE S(VILI) IN CERVICAL CANCER.
}

\author{
DR. ARUBA KHALID, MBBS \\ Nishtar Hospital Multan, Pakistan. \\ DR. AYESHA ASLAM, MBBS \\ Nishtar Hospital Multan, Pakistan. \\ DR. NIDA TARIQ KHAN, MBBS \\ Nishtar Hospital Multan, Pakistan.
}

\begin{abstract}
Introduction: Cervical cancer is the second most common gynecologic cancer worldwide, accounting for $13 \%$ of all female cancer in developing countries. Cervical cancer is a disease that can be prevented through both primary prevention and early detection. Pap smear is a screening method which has been used for so long. Hence, one simple diagnostic test namely visual inspection with Lugol 's iodine (VILI) which is based on the ability of the trained healthcare personnel to detect yellow non iodine uptake areas in the cervical transformation zone are currently being evaluated in the experimental setting as potential alternative to cervical cytology. Objective: To determine diagnostic accuracy of visual inspection with Lugol's iodine (VILI) taking histopathology as gold standard among patients of cervical cancer. Subjects and methods: A total of 285 women with early marriage, history of post-coital bleeding, history of post- menopausal bleeding and history of per vaginal discharge were included in study. Women with obvious cervical growth and diagnosed cervical cancer were excluded. I put each patient in the lithotomy position and insert Cusco's speculum to visualize cervix. I cleaned the cervix with cotton soaked with normal saline. Now applied Jugol 's iodine, to the cervix normal squamous epithelium contain glycogen and stain. Conversely premalignant and malignant squamous tissue contain no glycogen and does not stain with iodine. This was schillers test and non-staining areas were schiller positive. For further confirmation I took biopsy from schillers positive area and sent for histopathology. Results: Age range in this study was from 21 to 60 years with mean age of $39.393 \pm 6.66$ years and mean duration of disease was $6.031 \pm 2.09$ months. $43.9 \%$ patients were belong to rural area and $56.1 \%$ belongs to urban area. $25.6 \%$ patients have Family History of cervical cancer. Visual inspection with Lugol's iodine (VILI) diagnosed 43(15.1\%) patients and Histopathology diagnosed $15.8 \%$ patients with Cervical cancer. Visual inspection with Lugol's iodine (VILI) has shown sensitivity of $86.6 \%$, specificity $98.3 \%$, diagnostic accuracy by $96 \%$, PPV 90.6\%, NPV 97.5\%, ( $p=0.000)$ in diagnosis of Cervical cancer. Conclusion: My study showed VILI good sensitivity and it can therefore be a suitable potential alternative/adjunctive screening test not only in resource-poor settings but also in well-equipped centers.
\end{abstract}

Keywords: Cervical cancer, Visual inspection with Lugol's iodine, Diagnostic accuracy

DOI: $10.7176 / \mathrm{JMPB} / 52-07$

\section{INTRODUCTION}

Cervical cancer is the second most common gynecologic cancer worldwide, accounting for $13 \%$ of all female cancer in developing countries.(1) In India it is the second most common cancer in women aged 15-44 years. India has the highest age standardized incidence of cervical cancer in South Asia at 22, compared to 19.2 in Bangladesh, 13 in Sri Lanka, and 2.8 in Iran.(2) In Pakistan researched evidence puts 3.6\% of total female cancers to be of cervical origin, affecting women mainly in fifth and sixth decades of life.(3)

Despite of its public health importance there is no effective prevention program me in most of the developing countries and hence the risk of disease and death from cervical cancer remains largely uncontrolled. (4)

Cervical cancer is a disease that can be prevented through both primary prevention and early detection. So in developed countries the incidence of cervical cancer has decreased due to screening, early detection and treatment. However in developing countries, $80 \%$ of cervical cancers are incurable at the time of detection due to their advanced stage.(5)

Carcinoma of cervix, due to its slow progression from pre-cancerous lesion to malignancy and easy accessibility to examination, gives us ample opportunity for early detection and thus considerably improved prognosis. Early detection may be through opportunistic examination of women attending outpatient clinics or through systematic programme of screening. 
Pap smear is a screening method which has been used for so long.(6) The high incidence of cervical cancer i $n$ developing countries, represent that Pap smear screening test in the developing world was not effective.(7) Proper Pap smear test includes many steps which are not available in many countries.(8) Moreover, Pap tests themselves have short comings; high specificity in Pap smear testing cannot be achieved without reducing sensitivity.

Hence, one simple diagnostic test namely visual inspection with Lugol 's iodine (VILI) which is based on the ability of the trained healthcare personnel to detect yellow non iodine uptake areas in the cervical transformation zone are currently being evaluated in the experimental setting as potential alternative to cervical cytology.

In one study sensitivity of VILI in detecting cervical cancer was $89.50 \%$, specificity $75.90 \%$, positive predictive value $70.80 \%$ and negative predictive value $91.70 \%$. Prevalence of cervical cancer was $10 \% .(9,10)$

Considering the cervical cancer a life threatening problem, I have planned to conduct a study to determine the diagnostic accuracy of VI LI in detection of cervical cancer.

Cervical cancer: any one or more these; atypical tumor cells form ribbons, tubules or nests, broke the basement membrane of the duct and infiltrate the surrounding tissues or sometimes the tumor cells are arranged i n slender linear strands one to two cells across as assessed on histopathology.

Diagnostic Accuracy: Accuracy of the test was measured as the proportion of patients accurately diagnosed by VILI

VILI was considered positive on detection of iodine non-uptake in the transformation zone. And was labeled as YES.

\section{MATERIALS \& METHODS:}

Women with obvious cervical growth AND Women with diagnosed cervical cancer were excluded from our study. Patients came to the Outpatient and Emergency Department of Obstetrics and Gynaecology, Nishtar Hospital Multan fulfilling the inclusion and exclusion criteria were enrolled in the study. Researchers cleaned the cervix with cotton soaked with normal saline. Now applied Jugol 's iodine, to the cervix normal squamous epithelium contain glycogen and stain. Conversely premalignant and malignant squamous tissue contain no glycogen and does not stain with iodine. This was schillers test and non-staining areas were schiller positive. For further confirmation we took biopsy from schillers positive area and sent for histopathology. Data was entered and analysis by using SPSS software. Descriptive statistics were used to calculate mean and standard deviation of age of the patients and disease duration. Frequencies and percentages were calculated for age, residential status, family history, histopathology, VILI.

\section{RESULTS}

Age range in this study was from 21 to 60 years with mean age of $39.393 \pm 6.66$ years and mean duration of disease was $6.031 \pm 2.09$ months, $43.9 \%$ patients were belong to rural area and $56.1 \%$ belongs to urban area, $25.6 \%$ patients have Family History of cervical cancer. Visual inspection with Lugol's iodine (VILI) diagnosed 43(15.1\%) patients and Histopathology diagnosed $15.8 \%$ patients with Cervical cancer. Visual inspection with Lugol's iodine (VILI) has shown sensitivity of $86.6 \%$, specificity $98.3 \%$, diagnostic accuracy by $96 \%$, PPV 90.6\%, NPV 97.5\%, $(\mathrm{p}=0.000)$ in diagnosis of Cervical cancer.

\section{Discussion:}

Each year, about 490000 new cases of cervical cancer occurred worldwide, mainly in developing countries $(80 \%)$. In these countries, cervical cancer is the second most common cancer among women after breast cancer and the leading cause of cancer deaths. Such programs are not yet implemented in many resources constrained settings, where access to early detection of cervical neoplasia is still challenging and most patients are diagnosed at an advanced stage. Since 2005 the World Health Organization (WHO) recommended visual inspection of cervix with acetic acid (VIA) or Lugol's iodine (VILI) as alternative screening techniques for the detection of cervical precancerous lesions $(11,12)$. Many field evaluation surveys reported that visual inspection demonstrated diagnostic accuracy close to cytology and was more affordable and easy to perform for nonmedical health workers in developing countries. Côte d'Ivoire is now planning to implement a national cervical cancer screening program and the screening technic as well as the entry point to screen a maximum of women that are questions of interest.

In a meta analysis done by Fahey et al. in 1995(13) involving 62 studies conducted between 1984 and 1992, the mean sensitivity and specificity of cytology was 58\% (range 11-99\%) and 68\% (range 14-97\%), respectively. In another recent metaanalysis by Nanda et al. in 2000,(14) the sensitivity of cytology to the detection of CIN 2 or 
worse lesions ranged from $18 \%$ to $98 \%$ and the specificity ranged from $17 \%$ to $99 \%$. In the IARC multicenter study done in India and Africa by Sankaranarayanan et al. in 2004,(15) which included 11 cross-sectional studies, the sensitivity of VILI ranged from $56.10 \%$ to $93.90 \%$ and the specificity ranged between $74.20 \%$ and $93.80 \%$.

In the present study, Visual inspection with Lugol's iodine (VILI) has shown sensitivity of $86.6 \%$, specificity $98.3 \%$, diagnostic accuracy by $96 \%$, PPV 90.6\%, NPV 97.5\%, $(\mathrm{p}=0.000)$ in diagnosis of Cervical cancer. In the present study, the results are in comparison with that of the above-mentioned studies, suggesting that VILI may find a place as an alternative low-resource technology and low-cost method of screening and case finding. In one study sensitivity of VILI in detecting cervical cancer was $89.50 \%$, specificity $75.90 \%$, positive predictive value $70.80 \%$ and negative predictive value $91.70 \%$. Prevalence of cervical cancer was $10 \% .(9,10)$

In the IARC multicenter study done in India and Africa by Sankaranarayanan et al. in 2004,(15) which included 11 cross-sectional studies, the sensitivity of VILI ranges from $76.00 \%$ to $97.00 \%$ and the specificity between $73.00 \%$ and $91.30 \%$.

In the present study, of the 210 patients, VILI was positive in 24 (i.e., 11.43\%) patients. (Sensitivity $89.5 \%$ and specificity $75.90 \%$ ). In our study, the reason for high sensitivity and low percentage of FP results of VILI are because Lugol's iodine produces characteristic nonuptake yellow areas in suspected cases that are easy to interpret, and observation was based on the well-defined criteria to identify between iodine uptake and nonuptake, mahagony brown area versus yellow areas. An atlas also has been referred from time to time for characteristic identification of lesions in case of doubt.The Sankarnarayan et al. 2004(15) study showed a similar result and recommended the use of both VILI in parallel to increase test sensitivity.

Our results are consistent with that of the Panten et al. 1995(16) study at the University of Jimbanbe/JHPIEGO cervical cancer project 1999,(17) Denny et al. 2000(18) and Shankaranarayan et al. 2003(19) studies, which showed that combining VIA and VILI with Pap smear markedly improved their performance as screening tests at the cost of large number of women being referred for further treatment in view of false-positive results and decreased specificity of the test.

An attempt has been made in present study to increase awareness of women about cervical cancer and preventive health-seeking behavior, screen all women of the reproductive age group at least once a year and motivate them for annual screening until three negative Pap smears, to provide a screening test with high sensitivity as women have less frequent opportunities for repeated screening and treating women with high-grade dysplasia and cancer.

Women continue to ignore symptoms of irregular bleeding, postmenopausal bleeding and postcoital bleeding. Therefore, in our study, we aimed at educating women about these signs and symptoms and to seek immediate medical care. Thus, an attempt has been made to target the disease before its onset at the level of primary prevention by providing education and counseling and secondary prevention by effective screening and treatment.

\section{Conclusion:}

Cervical cancer is "preventable but not prevented" remains the reality today, because no perfect screening tests are available that have $100 \%$ sensitivity and good specificity. Therefore, in the present study, an attempt has been made to analyze VILI as standalone test.

My study showed VILI good sensitivity and it can therefore be a suitable potential alternative/adjunctive screening test not only in resource-poor settings but also in well-equipped centers.

\section{REFERENCES:}

1. Ferlay J, Shin HR, Bray F, Forman D, Mathers C, Parkin OM. Estimates of worldwide burden of cancer in 2008. GLOBOCAN 20 Int J Cancer 2010;127:2893-917.

2 . Sreedevi A, Javed R, Dinesh A. Epidemiology of cervical cancer with special focus on India. Int J Womens Health. 2015;7:405-14.

3. Abbasi AN, Zahid S, Bhurgri Y, Ali N, Karsan F. Nasopharyngeal carcinoma - an u pdate of treatment and acute radiation induced reactions from a tertiary-care hospital in Pakistan. Asian Pac J Cancer Prev 201 $1 ; 12: 735-8$.

4. Bhattacharyya AK, Nath JD, Deka H. Comparative study between pap smear and visual inspection with acetic acid (via) in screening of CIN and early cervical cancer. J Midlife Health. 2015;6(2):53-8.

5. Bryan JT, Buckland B, Hammond J, Jansen KU. Prevention of cervical cancer : journey to develop the first human papillomavirus virus-like pa11iclevaccine and the next generation vaccine. Curr Opin Chem Biol. 2016 Jun;32:34-47. 
6. Gaffikin L, McGrath JA, Arbyn M, Blu menthal PD. Visual inspection with acetic acid as a cervical cancer test: accuracy val idated using latent class analysis. BMC Med Res Methodol. 2007;7:36.

7. Catarino R, Petignat P, Dongui G, Vassilakos P. Cervical cancer screening in developing countries at a crossroad: Emerging technologies and policy choices. World J Clin Oncol. 2015;6(6):281-90.

8. Selmouni F, Sauvaget C, Zidouh A, Plaza CA, Muwonge R, Rhazi KE, et al. Evaluation of Provider Skills in Performing Visual Inspection with Acetic Acid in the Cervical Cancer Screening Program in the MeknesTafilalet Region of Morocco. Asian Pac J Cancer Prev. 2016;17(9):4313-4318.

9. Consul S, Agrawal A, Sharma H, Bansal A, Gutch M, Jain N. Comparative study of effectiveness of Pap smear versus visual inspection with acetic acid and visual inspection with Lugol's iodine for mass screening of premalignant and malignant lesion of cervix. Indian J Med Paediatr Oncol. 2012;33(3):161-5..

10. Ferlay J, Shin HR, Bray F, Forman D, Mathers C, Parkin DM. Estimates of worldwide burden of cancer in 2008: GLOBOCAN 2008. Int J Cancer. 2010 Dec 15;127(12):2893-917.

11. Sankaranarayanan R, Wesley R. Visual inspection of the uterine cervix with acetic acid (VIA) and Lugol's iodine (VILI)- A technical manual of IARC, 1999.

12. Sellors JW, Jeronimo J, Sankaranarayanan R, Wright TC, Howard M, Blumenthal PD. Assessment of cervix after acetic acid wash: inter-rater agreement using photographs. Obstetrics and Gynecology 2002, 99:635-40.

13 Fahey MT, Irwig L, Macaskill P. Meta-analysis of Pap test accuracy. Am J Epidemiol. 1995;141:680-9.

14. Nanda K, McCrory DC, Myers ER, Bastian LA, Hasselblad V, Hickey JD, et al. Accuracy of the Papanicolaou test in screening for and followup of cervical cytologic abnormalities: a systematic review. Ann Intern Med. 2000;132:810-9.

15. Sankaranarayanan R, Basu P, Wesley RS, Mahé C, Keita N, Mbalawa CC, et al. Accuracy of visual screening for cervical neoplasia: Results from an IARC multicentre study in India and Africa. Int J Cancer. 2004;110:907-13.

16. Panten J, Adami HO, Bergstrom R, Dillner J, Friberg LG, Gustafsson L, et al. Strategies for Global Control of Cervical Cancer. Int J Cancer. 1995;60:1-26.

17. University of Zimbabwe/JHPIEGO Cervical Cancer Project Visual inspection with acetic acid for cervicalcancer screening: test qualities in a primary-care setting. Lancet. 1999;353:869-73.

18. Denny L, Kuhn L, Pollack A, Wainwright H, Wright TC., Jr Evaluation of alternative methods of cervical cancer screening for resource-poor settings. Cancer. 2000;89:826-33.

19. Sankaranarayanan R, Nene BM, Dinshaw K, Rajkumar R, Shastri S, Wesley R, et al. Early detection of cervical cancer with visual inspection methods: a summary of completed and on-going studies in India. Salud Publica Mex. 2003;45(Suppl 3):S399-407. 